Asia Pacific Journal of Mathematics, Vol. 5, No. 1 (2018), 27-49

ISSN 2357-2205

\title{
MULTIPLICITY OF SOLUTIONS TO DISCRETE INCLUSIONS WITH THE $p(k)$-LAPLACE KIRCHHOFF TYPE EQUATIONS
}

\author{
STANISLAS OUARO*, MALICK ZOUNGRANA
}

Université Ouaga I Pr Joseph KI-ZERBO, Unité de Formation et de Recherche en Sciences Exactes et Appliquées, Département de Mathématiques

B.P. 7021 Ouagadougou 03, Burkina Faso

*Corresponding author: souaro@univ-ouaga.bf, ouaro@yahoo.fr

Received Oct 4, 2017

\begin{abstract}
This paper is concerned with the existence and multiplicity of solutions to discrete inclusions with an anisotropic discrete boundary value problem of $p(k)$-Laplace Kirchhoff type. Our technical approach is based on variational methods.

2010 Mathematics Subject Classification.47A75, 35B38, 35P30, 34L05, 34L30.

Key words and phrases.Kirchhoff type equation; multiple solutions; discrete inclusion; discrete boundary value problem; critical point; variational methods.
\end{abstract}

\section{Introduction}

In this work, we study the existence and multiplicity of solutions to discrete inclusions of the following Kirchhoff type problem

$$
\left\{\begin{array}{l}
-M(A(k-1, \Delta u(k-1))) \Delta(a(k-1, \Delta u(k-1))) \in \lambda \partial F(k, u(k)), \quad k \in \mathbb{Z} \\
u(k+m)=u(k), \forall k \in \mathbb{Z},
\end{array}\right.
$$

where $\Delta u(k)=u(k+1)-u(k)$ is the forward difference operator, $u(k) \in \mathbb{R}^{n}$ for all $k \in \mathbb{Z}$; $a(k,):. \mathbb{R}^{n} \rightarrow \mathbb{R}^{n}$ is a continuous function for all $k \in \mathbb{Z}$ and there exists a mapping $A$ : $\mathbb{Z} \times \mathbb{R}^{n} \longrightarrow \mathbb{R}^{n}$ satisfying $a(k, \xi)=\frac{\partial A(k, \xi)}{\partial \xi}, \forall k \in \mathbb{Z}$ and $A(k, 0)=0$ for all $k \in \mathbb{Z}$. Let the function $F: \mathbb{Z} \times \mathbb{R}^{n} \longrightarrow \mathbb{R}^{n}$ and $\partial F(k, u)$ denotes the Clarke subdifferential of $F$ with respect to the second variable. Recall that if $f: X \longrightarrow \mathbb{R}^{n}$ is a locally Lipschitz functional and $x \in X$, the Clarke subdifferential of $f$ at the point $x$ is the nonempty subset $\partial f(x)$ of 
$X^{*}$ which is defined by

$$
\partial f(x):=\left\{x^{*} \in X^{*}:\left\langle x^{*}, v\right\rangle \leq f^{0}(x ; v), \text { for all } v \in X\right\}
$$

where $f^{0}(x ; v)$ is the generalized directional derivative of $f$ at the point $x$ along the direction $v$ (to be defined later).

Here, $\lambda$ is a positive real parameter and $m \geq 2$ is a fixed natural number.

$M(t)$ is a continuous function that satisfy some conditions which will be stated later on.

Here, we are interested in investigating nonlinear discrete boundary value problems by using variational approach. We refer to $[1,2,3,4,5,6,14,15,18,19,20,21,27,29]$ and the references therein for more details about discrete boundary value problems.

Problem (1.1) has its origin in the theory of non linear vibration. For instance, the following equation describes the free vibration of a stretched string (see [24])

$$
\rho \frac{\partial^{2} u}{\partial t^{2}}=\left(T_{0}+\frac{E a}{2 L} \int_{0}^{L}\left|\frac{\partial u}{\partial x}\right|^{2} d x\right) \frac{\partial^{2} u}{\partial x^{2}}
$$

where $\rho>0$ is the mass per unit length, $T_{0}$ is the base tension, $E$ is the Young modulus, $a$ is the area of cross section and $L$ is the initial length of the string.

Equation (1.2) takes into account the change of the tension on the string which is caused by the change of its length during the vibration. The nonlocal equation of this type was firstly proposed by Kirchhoff in 1876 (see [16]). After that, several physicists also considered such equations for their researches in the theory of nonlinear vibrations theoretically or experimentally $[7,8,24,25]$. As far as we know, the first study which deals with anisotropic discrete boundary-value problems of $p($.)-Kirchhoff type difference equation was done by Yucedag (see [28]). A more general study of the problem of Yucedag has been done by Koné et al (see [17]). In this paper our aim is to establish the existence and multiplicity results for problem (1.1) through variational methods.

We will use some abstract tools contained in $[23,26]$ studying the above anisotropic discrete inclusions.

The remaining part of this article is organized as follows: some usefull preliminary results are presented in Section 2. In Section 3, we recall the new abstract critical point theorems established in [11], while Section 4 is devoted to the multiplicity results for problem (1.1).

\section{Basic definitions and preliminary results}

Let $(E,\|\|$.$) be a real Banach space. We denote by E^{*}$ the dual space of $E$, while $\langle$,$\rangle stands$ for the duality pairing between $E^{*}$ and $E$.

A function $J: E \longrightarrow \mathbb{R}$ is called locally Lipschitz continuous, if for every $u \in E$, there exist 
a neighbourhood $V_{u}$ of $u$ and a constant $L_{u} \geq 0$ such that

$$
|J(z)-J(w)| \leq L_{u}\|z-w\| \text { for all } z, w \in V_{u}
$$

If $u, z \in E$, we write $J^{0}(u ; z)$ for the generalized directional derivative of $J$ at the point $u$ along the direction $z$, i.e.

$$
J^{0}(u ; z):=\lim _{w \rightarrow u} \sup _{t \rightarrow 0^{+}} \frac{J(w+t z)-J(w)}{t} .
$$

The generalized gradient of the function $J$ in $u$, denoted by $\partial J(u)$, is the set

$$
\partial J(u):=\left\{u^{*} \in E^{*}:\left\langle u^{*}, z\right\rangle \leq J^{0}(u ; z) \text {, for all } z \in E\right\} .
$$

The basic properties of generalized directional derivative and generalized gradient were studied in $[9,10]$.

We recall that if $J$ is continuously Gâteaux differentiable at $u$, then $J$ is locally Lipschitz at $u$ and $\partial J(u)=\left\{J^{\prime}(u)\right\}$, where $J^{\prime}(u)$ stands for the first derivative of $J$ at $u$.

Furthermore, a point $u$ is called a (generalized) critical point of the locally Lipschitz continuous function $J$, if $0_{E^{*}} \in \partial J(u)$, i.e.

$$
J^{0}(u ; z) \geq 0, \text { for every } z \in E .
$$

Clearly, if $J$ is continuously Gâteaux differentiable at $u$, then $u$ becomes a (classical) critical point of $J$, that is $J^{\prime}(u)=0_{E^{*}}$.

A locally Lipschitz continuous functional $J: E \longrightarrow \mathbb{R}$ is said to fulfil the Palais-Smale (PS) condition if every sequence $\left\{u_{n}\right\}$ in $E$ such that $\left\{J\left(u_{n}\right)\right\}$ is bounded and

$$
J^{0}\left(u_{n} ; u-u_{n}\right) \geq-\epsilon_{n}\left\|u-u_{n}\right\|
$$

for all $u \in E$, where $\epsilon_{n} \longrightarrow 0^{+}$as $n \longrightarrow \infty$, possesses a convergent subsequence.

Definition 2.1. A function $f$ defined on a normed space $X$ to $\overline{\mathbb{R}}$ is said to be coercive over an unbounded part $P$ of $X$ if $\lim _{\|x\| \longrightarrow+\infty} f(x)=+\infty$, where $x \in P$. $f$ is said to be anti-coercive if $(-f)$ is coercive.

For a complete overview on the non-smooth calculus we refer the readers to the paper [22]. Furthermore, let's cite a recent book [19] as a general reference on the subject of our paper. Our main tool will be the following abstract critical point theorems, for locally Lipschitz continuous functions that we recall here, for completeness, in their general form. 
Theorem 2.2. ([26], Theorem 2.3) Let $E$ be a real Banach space. Assume that $E:=E_{1} \oplus E_{2}$, with $E_{2}$ finite-dimensional subspace of $E$. Let $J: E \longrightarrow \mathbb{R}$ be a locally Lipschitz continuous functional satisfying the (PS) condition and such that

$J(u) \leq 0,\left(\forall u \in \bar{B}(0, \rho) \cap E_{2}\right)$,

$J(u) \geq 0,\left(\forall u \in \bar{B}(0, \rho) \cap E_{1}\right)$,

for some $\rho>0$.

Assume also that $J$ is bounded from below and $\inf _{u \in E} J(u)<0$. Then, $J$ has at least two non-zero critical points.

Theorem 2.3. [23] Let $(E,\|\|$.$) be a real Banach space and let J: E \longrightarrow \mathbb{R}$ be a locally Lipschitz continuous functional satisfying $(P S)$ condition. If there exist $u_{1}, u_{2} \in E, u_{1} \neq u_{2}$ and $r \in\left(0,\left\|u_{2}-u_{1}\right\|\right)$ such that inf $\left\{J(u):\left\|u-u_{1}\right\|=r\right\} \geq \max \left\{J\left(u_{1}\right), J\left(u_{2}\right)\right\}$ and we denote by $\Gamma$ the family of continuous paths $\gamma:[0,1] \longrightarrow E$ joining $u_{1}$ and $u_{2}$, then

$c:=\inf _{\gamma \in \Gamma} \max _{s \in[0,1]} J(\gamma(s)) \geq \max \left\{J\left(u_{1}\right), J\left(u_{2}\right)\right\}$ is a critical value for $E$ and $K_{c}-\left\{u_{1}, u_{2}\right\} \neq \emptyset$, where $K_{c}$ is the set of critical points at the level $c$.

We now make the following assumptions on the data.

$\left(H_{1}\right): \exists A: \mathbb{Z} \times \mathbb{R}^{n} \rightarrow \mathbb{R}^{n}$ with $a(k, \xi)=\frac{\partial A(k, \xi)}{\partial \xi}, \forall k \in \mathbb{Z}, \xi \in \mathbb{R}^{n}$ and $A(k, 0)=0$ for all $k \in \mathbb{Z}$.

$\left(H_{2}\right): \exists C_{1}>0$ such that $|a(k, \xi)| \leqslant C_{1}\left(1+|\xi|^{p(k)-1}\right), \quad \forall k \in \mathbb{Z}$ and $\forall \xi \in \mathbb{R}^{n}$.

$\left(H_{3}\right):(a(k, \xi)-a(k, \eta)) \cdot(\xi-\eta)>0, \forall(\xi, \eta) \in \mathbb{R}^{n} \times \mathbb{R}^{n}$ such that $\xi \neq \eta$ and $\forall k \in \mathbb{Z}$.

$\left(H_{4}\right):|\xi|^{p(k)} \leq a(k, \xi) \cdot \xi \leq p(k) A(k, \xi), \xi \in \mathbb{R}^{n}$ and $p: \mathbb{Z} \rightarrow(2,+\infty)$, where $p$ is an $m$-periodic function, i.e. $p(k+m)=p(k), \forall k \in \mathbb{Z}$.

$\left(H_{5}\right): a$ is an $m$-periodic function with respect to $k$, i.e. $a(m+k, \xi)=a(k, \xi)$ for all $(k, \xi) \in$ $\mathbb{Z} \times \mathbb{R}^{n}$.

$\left(H_{6}\right): F$ is an $m$-periodic function with respect to $k$, i.e. $F(k, u)=F(k+m, u)$ for all $(k, u) \in \mathbb{Z} \times \mathbb{R}^{n}$.

$\left(H_{7}\right): F(k,$.$) is locally Lipschitz continuous for all k \in \mathbb{Z}$.

$\left(H_{8}\right): F(k, 0)=0$ for all $k \in \mathbb{Z}$.

$\left(H_{9}\right): M:(0,+\infty) \longrightarrow(0,+\infty)$ is continuous and nondecreasing and there exist positive reals $B_{1}, B_{2}$ with $B_{1} \leq B_{2}$ and $\alpha>1$ such that

$$
B_{1} t^{\alpha-1} \leq M(t) \leq B_{2} t^{\alpha-1} \text { for } t \geq t^{*}>0
$$


Example 2.4. As examples of functions satisfying assumptions $\left(H_{1}\right)-\left(H_{9}\right)$, we can give the following.

(1) $M(A(k, \xi))=\frac{1}{p(k)}|\xi|^{p(k)}=1$, where $M(t)=1$ and $a(k, \xi)=|\xi|^{p(k)-2} \cdot \xi$, for $k \in \mathbb{Z}$ and $\xi \in \mathbb{R}^{n}$.

(2) $M(A(k, \xi))=a+\frac{b}{p(k)}\left[\left(1+|\xi|^{2}\right)^{\frac{p(k)}{2}}-1\right]$, where $M(t)=a+b t$ and $a(k, \xi)=(1+$ $\left.|\xi|^{2}\right)^{(p(k)-2) / 2} . \xi$, for $k \in \mathbb{Z}$ and $\xi \in \mathbb{R}^{n}$.

(3) $F: \mathbb{Z} \times \mathbb{R}^{n}$ given by

$$
F(k, t)\left\{\begin{array}{l}
16 t^{4} \text { if }|t| \leq \frac{1}{2} \\
-4|t|+3 \text { if }|t| \in\left(\frac{1}{2}, 1\right) \\
t^{4}+|t-1|-2 \text { if }|t| \geq 1
\end{array}\right.
$$

\section{Three critical points theorem for locally Lipschitz functionals}

In this section we recall the tools used in [11].

Theorem 3.1. Let $(X, \tau)$ be an Hausdorff space and $\Phi, J: X \longrightarrow \mathbb{R}$ be functionals. Let $M$ be the set (possibly empty) of all the global minimizers of $J$ and define

$$
\begin{gathered}
\alpha:=\inf _{x \in X} \Phi(x), \\
\beta:= \begin{cases}\inf _{x \in M} \Phi(x) & \text { if } M \neq \emptyset \\
\sup _{x \in X} \Phi(x) & \text { if } M=\emptyset .\end{cases}
\end{gathered}
$$

Let $\alpha<\beta$ and assume that the set

$$
\{x \in X: \Phi(x)+\sigma J(x) \leq \rho\}
$$

for every $\sigma>0$ and every $\rho \in \mathbb{R}$, is sequentially compact (if not empty). Then at least one of the following conditions holds.

(a) There exists a continuous mapping $h:(\alpha, \beta) \longrightarrow X$ with the following property: for every $t \in(\alpha, \beta)$,

$$
\Phi(h(t))=t
$$

and for every $x \in \Phi^{-1}(t)$ with $x \neq h(t)$,

$$
J(x)>J(h(t)) .
$$


(b) There exists $\lambda>0$ such that the functional $\Phi+\lambda J$ admits at least two global minimizers in $X$.

Theorem 3.2. Let $E$ be a finite dimensional real Banach space. Let $\mu: E \longrightarrow \mathbb{R}$ be a coercive $C^{1}$ functional such that $\mu(0)=0$ and let $J: E \longrightarrow \mathbb{R}$ be locally Lipschitz. Let $s>0$ and $0<r<s$ be fixed. Assume that

$\left(b_{1}\right) \lim _{\mu(u) \longrightarrow+\infty} \inf \frac{J(u)}{\mu(u)} \geq 0$

$\left(b_{2}\right) \inf _{u \in E} J(u)<\inf _{\mu(u) \leq s} J(u)$

$\left(b_{3}\right) J(0) \leq \inf _{r \leq \mu(u) \leq s} J(u)$.

Then, there exists $\lambda>0$ such that the functional $\mu+\lambda J$ has at least three critical points in $E$, at least two of which are non-trivial.

Theorem 3.3. Let $E$ be a finite dimensional real Banach space. Let $\mu: E \longrightarrow \mathbb{R}$ be a coercive $C^{1}$ functional such that $\mu(0)=0$ and let $J: E \longrightarrow \mathbb{R}$ be a locally lipschitz functional bounded from below. Let $s>0$ and $0<r<s$ be fixed constants. Assume moreover that conditions $\left(b_{2}\right)$ and $\left(b_{3}\right)$ hold. Then there exists $\lambda>0$ such that the functional $\mu+\lambda J$ has at least three critical points in $E$.

\section{Variational framework and auxiliary results}

From now on, we will use the following notations.

$$
p^{+}:=\max _{k \in \mathbb{Z}[1, m]} p(k) \text { and } p^{-}:=\min _{k \in \mathbb{Z}[1, m]} p(k),
$$

where $\mathbb{Z}[a, b]:=\{a, a+1, \ldots, b\}$, with $a, b \in \mathbb{N}$ such that $a \leq b$.

Define the space

$$
H_{m}=\left\{u=\{u(k)\}_{k \in \mathbb{Z}}: u(k) \in \mathbb{R}^{n}, u(k+m)=u(k), k \in \mathbb{Z}\right\},
$$

which equipped with the Euclidean norm

$$
\|u\|_{e}:=\left(\sum_{k=1}^{m}|u(k)|^{2}\right)^{\frac{1}{2}}
$$

becomes an Hilbert space.

Put for any $a \in \mathbb{R}^{n}$, 
$W_{a}:=\left\{u=\{u(k)\}_{k \in \mathbb{Z}}: u(k)=a, k \in \mathbb{Z}\right\}$ and $Y_{a}:=W_{a}^{\perp}$.

Thus, $W_{a}$ consists of constant sequences and we have an orthogonal decomposition

$$
H_{m}=Y_{a} \oplus W_{a} \text {, for any } a \in \mathbb{R}^{n} .
$$

The energy functional corresponding to (1.1) is

$$
J_{m}(u)=\widehat{M}\left(\sum_{k=1}^{m} A(k-1, \Delta u(k-1))\right)-\lambda \sum_{k=1}^{m} F(k, u(k))
$$

where $\widehat{M}(t)=\int_{0}^{t} M(s) d s$.

Definition 4.1. A solution of problem (1.1) is a function $u \in H_{m}$ such that

$-M\left(\sum_{k=1}^{m} A(k-1, \Delta u(k-1))\right) \sum_{k=1}^{m} \Delta(a(k-1 ; \Delta u(k-1))) h(k)-\lambda \sum_{k=1}^{m} F^{0}(k ; u(k)) h(k) \geq 0$,

for all $h \in H_{m}$ with $h(k) \geq 0$ and for all $k \in \mathbb{Z}$.

Proposition 4.2. Assume that $\left(H_{1}\right),\left(H_{2}\right),\left(H_{6}\right)$ and $\left(H_{9}\right)$ hold. Then $J_{m}$ is locally Lipschitz continuous.

\section{Proof.}




$$
\begin{aligned}
& |J(z)-J(w)|=\mid \widehat{M}\left(\sum_{k=1}^{m} A(k-1, \Delta z(k-1))\right)-\widehat{M}\left(\sum_{k=1}^{m} A(k-1, \Delta w(k-1))\right) \\
& -\lambda \sum_{k=1}^{m} F(k, z(k))+\lambda \sum_{k=1}^{m} F(k, w(k)) \\
& \leq\left|\widehat{M}\left(\sum_{k=1}^{m} A(k-1, \Delta z(k-1))\right)-\widehat{M}\left(\sum_{k=1}^{m} A(k-1, \Delta w(k-1))\right)\right| \\
& +\lambda \sum_{k=1}^{m}|F(k, z(k))-F(k, w(k))| \\
& =\left|\int_{\sum_{k=1}^{m} \sum_{k=1}^{m} A(k-1, \Delta w(k-1))}^{m} M(t) d t\right|+\lambda \sum_{k=1}^{m}|F(k, z(k))-F(k, w(k))| \\
& \leq \int_{\sum_{k=1}^{m} A(k-1, \Delta w(k-1))}^{\sum_{k=1}^{m} A(k-1, \Delta z(k-1))} B_{2} t^{\alpha-1} d t+\lambda \sum_{k=1}^{m}|F(k, z(k))-F(k, w(k))| \\
& \leq \frac{B_{2}}{\alpha}\left[\left(\sum_{k=1}^{m} A(k-1, \Delta z(k-1))\right)^{\alpha}-\left(\sum_{k=1}^{m} A(k-1, \Delta w(k-1))\right)^{\alpha}\right] \\
& +\lambda \sum_{k=1}^{m}|F(k, z(k))-F(k, w(k))| \text {. }
\end{aligned}
$$

If $w=z$ then, $J(z)=J(w)$. Consequently, $\exists K_{1}>0$ such that $|J(z)-J(w)| \leq K_{1}\|z-w\|_{e}$. If $z \neq w$ then, $\|w-z\| \neq 0$ and since the quantity $\left[\left(\sum_{k=1}^{m} A(k-1, \Delta z(k-1))\right)^{\alpha}-\left(\sum_{k=1}^{m} A(k-\right.\right.$ $\left.1, \Delta w(k-1)))^{\alpha}\right]$ is finite then, $\exists K_{2}>0$ such that

$$
\left[\left(\sum_{k=1}^{m} A(k-1, \Delta z(k-1))\right)^{\alpha}-\left(\sum_{k=1}^{m} A(k-1, \Delta w(k-1))\right)^{\alpha}\right] \leq K_{2}\|z-w\|_{e}
$$

Consequently, since $F(k,$.$) is locally Lipschitz continuous then for all u \in H_{m}$, there exist a neighbourhood $V_{u}$ of $u$ and constant $C_{2} \geq 0$ such that $|F(k, z(k))-F(k, w(k))| \leq C_{2}\|z-w\|_{e}$. 
We can deduce that

$$
\begin{aligned}
|J(z)-J(w)| & \leq \frac{B_{2}}{\alpha} K_{2}\|z-w\|_{e}+\lambda m C_{2}\|z-w\|_{e} \\
& \leq L\|z-w\|_{e}, \text { with } L=\frac{B_{2}}{\alpha} K_{2}+\lambda m C_{2} .
\end{aligned}
$$

Lemma 4.3. Let $u \in H_{m}$ be a critical point of $J_{m}$. Then, u satisfies problem (1.1).

\section{Proof.}

Assume that $u \in H_{m}$ is a critical point of $J_{m}$. Then, for any $h \in H_{m}, J^{0}(u ; h) \geq 0$.

We have

$$
\begin{aligned}
J(w+t h) & =\widehat{M}\left(\sum_{k=1}^{m} A(k-1, \Delta(w+t h)(k-1))\right)-\lambda \sum_{k=1}^{m} F(k,(w+t h)(k)) \\
& =\widehat{M}\left(\sum_{k=1}^{m} A(k-1, \Delta w(k-1)+t \Delta h(k-1))\right)-\lambda \sum_{k=1}^{m} F(k, w(k)+t h(k)) ;
\end{aligned}
$$

consequently

$$
\begin{aligned}
J(w+t h)-J(w) & =\widehat{M}\left(\sum_{k=1}^{m} A(k-1, \Delta w(k-1)+t \Delta h(k-1))\right)-\widehat{M}\left(\sum_{k=1}^{m} A(k-1, \Delta w(k-1))\right) \\
& -\lambda \sum_{k=1}^{m} F(k, w(k)+t h(k))+\lambda \sum_{k=1}^{m} F(k, w(k)) \\
& =\widehat{M}\left(\sum_{k=1}^{m} A(k-1, \Delta w(k-1)+t \Delta h(k-1))\right)-\widehat{M}\left(\sum_{k=1}^{m} A(k-1, \Delta w(k-1))\right) \\
& -\lambda\left[\sum_{k=1}^{m} F(k, w(k)+t h(k))-\sum_{k=1}^{m} F(k, w(k))\right] .
\end{aligned}
$$

By dividing by $t$ and by making $t$ tend towards 0 and $w$ towards $u$, we obtain

$J^{0}(u ; h)=M\left(\sum_{k=1}^{m} A(k-1, \Delta u(k-1))\right) \sum_{k=1}^{m} a(k-1 ; \Delta u(k-1)) \Delta h(k-1)-\lambda \sum_{k=1}^{m} F^{0}(k ; u(k)) h(k)$.

Using Abel's summation by parts formula we get

$$
\begin{aligned}
\sum_{k=1}^{m} a(k-1 ; \triangle u(k-1)) \triangle h(k-1) & =\sum_{k=1}^{m} a(k-1 ; \triangle u(k-1)) h(k)-\sum_{k=1}^{m} a(k-1 ; \triangle u(k-1) h(k-1) \\
& =\sum_{k=1}^{m} a(k-1 ; \triangle u(k-1)) h(k)-\sum_{k=0}^{m-1} a(k ; \triangle u(k)) h(k) \\
& =\sum_{k=1}^{m} a(k-1 ; \triangle u(k-1)) h(k)-\sum_{k=1}^{m} a(k ; \triangle u(k)) h(k) \\
& -a(0, \triangle u(0)) h(0)+a(m, \triangle u(m)) h(m) .
\end{aligned}
$$


According to $\left(H_{5}\right)$ and as $h \in H_{m}$, it follows that

$$
\sum_{k=1}^{m} a(k-1 ; \Delta u(k-1)) \Delta h(k-1)=-\sum_{k=1}^{m} \Delta(a(k-1 ; \Delta u(k-1))) h(k)
$$

and finally $J^{0}(u ; h) \geq 0$ train $-M\left(\sum_{k=1}^{m} A(k-1, \Delta u(k-1))\right) \sum_{k=1}^{m} \Delta(a(k-1 ; \Delta u(k-1)) h(k)-$ $\lambda \sum_{k=1}^{m} F^{0}(k ; u(k)) h(k) \geq 0$

This means that $0_{H_{m}^{*}} \in \partial J(u)$ and thus $u$ satisfies (1.1).

Now, we recall some auxiliary results, which we use later on, see [12].

Lemma 4.4. The following properties hold.

$\left(a_{1}\right)$ For every $s>0$,

$$
\sum_{k=1}^{m}|u(k)|^{s} \leqslant m\|u\|_{e}^{s}, \text { for all } u \in H_{m}
$$

$\left(a_{2}\right)$ For every $s \geqslant 2$,

$$
\sum_{k=1}^{m}|u(k)|^{s} \geqslant m^{\frac{(2-s)}{2}}\|u\|_{e}^{s}, \text { for all } u \in H_{m}
$$

$\left(a_{3}\right)$ For all $u \in H_{m}$

$$
\sum_{k=1}^{m}|\triangle u(k-1)|^{p(k-1)} \leqslant m\left(2^{p^{+}}\|u\|_{e}^{p^{+}}+1\right) .
$$

\section{Multiple solutions of problem (1.1) by applying Theorem 2.2}

Assume that $F$ satisfies additionally the following.

$\left(a_{4}\right)$ There exist $m$-periodic functions $s: \mathbb{Z} \longrightarrow[2 ;+\infty), \alpha_{1}: \mathbb{Z} \longrightarrow(0,+\infty)$ and a function $\alpha_{2}: \mathbb{Z} \longrightarrow \mathbb{R}$ for which

$$
F(k, u) \geq \alpha_{1}(k)|u|^{s(k)}+\alpha_{2}(k)
$$

for all $k \in \mathbb{Z}$ and $u \in \mathbb{R}^{n}$ such that $|u| \geq S$, where $S \geq 1$ is fixed and sufficiently large.

Put $s^{-}:=\min _{k \in \mathbb{Z}[1, m]} s(k) ; \quad \alpha_{1}^{-}:=\min _{k \in \mathbb{Z}[1, m]} \alpha_{1}(k) ; \alpha_{2}^{-}:=\min _{k \in \mathbb{Z}[1, m]} \alpha_{2}(k)$, where $s, \alpha_{1}, \quad \alpha_{2}$ are functions defined above.

Assume further that 
$\left(a_{5}\right) \lim _{|u| \rightarrow 0} \frac{F(k, u)}{|u|^{s^{-}}}=0$ uniformly in $k \in \mathbb{Z}$.

$\left(a_{6}\right)$ There exist constants $\omega_{0}, \omega_{1}, \omega_{2}>0$, with $\omega_{2}>\omega_{1}>\omega_{0}$, such that for all $k \in \mathbb{Z}$ :

$\left(a_{6}^{\prime}\right) F(k, u) \geq 0$ with $|u| \leq \omega_{0}$;

$\left(a_{6}^{\prime \prime}\right) F(k, u)<0$ with $\omega_{1} \leq|u| \leq \omega_{2}$.

Example 5.1. As examples of functions satisfying assumptions $\left(H_{6}\right)-\left(H_{8}\right)$ and $\left(a_{4}\right)-\left(a_{6}\right)$ we can give the following, where $m \geq 2$ is a fixed even natural number.

(1) $F: \mathbb{Z} \times \mathbb{R}^{n}$ given by

$$
F(k, t):=\left\{\begin{array}{l}
16 t^{4} \text { if }|t| \leq \frac{1}{2} \\
-4|t|+3 \text { if }|t| \in\left(\frac{1}{2}, 1\right) \\
t^{4}+|t-1|-2 \text { if }|t| \geq 1
\end{array}\right.
$$

and

(2) $s: \mathbb{Z} \rightarrow[2,+\infty)$ such that

$$
s(k):=\left\{\begin{array}{l}
4 \text { if } k=2 l, \\
2 \text { if } k=2 l+1 ; l \in \mathbb{Z} .
\end{array}\right.
$$

Lemma 5.2. Assume that conditions $\left(H_{1}\right),\left(H_{2}\right),\left(H_{9}\right)$ and $\left(a_{4}\right)$ hold with $s^{-}>\alpha p^{+}$. Then, the functional $J_{m}$ is anti-coercive on $H_{m}$, for all $\lambda>0$.

\section{Proof.}

$J_{m}(u)=\widehat{M}\left(\sum_{k=1}^{m} A(k-1, \Delta u(k-1))\right)-\lambda \sum_{k=1}^{m} F(k, u(k))$.

One can use $\left(H_{1}\right)$ to say that

$$
A(k, \xi)=\int_{0}^{\xi} a(k, \lambda) d \lambda
$$

Using $\left(H_{2}\right)$, we have the existence of a real $C_{1}>0$ such that

$$
|a(k, \xi)| \leqslant C_{1}\left(1+|\xi|^{p(k)-1}\right) \quad \text { for all } \quad k \in \mathbb{Z} \quad \text { and for all } \quad \xi \in \mathbb{R}^{n} .
$$

Therefore,

$$
\begin{aligned}
\int_{0}^{\xi}|a(k, \lambda)| d \lambda & \leq C_{1} \int_{0}^{\xi}\left(1+|\lambda|^{p(k)-1}\right) d \lambda \\
& \leq C_{1}[\lambda]_{0}^{\xi}+C_{1}\left[\frac{\lambda^{p(k)}}{p(k)}\right]_{0}^{\xi} \\
& \leq C_{1}|\xi|+C_{1} \frac{|\xi|^{p(k)}}{p(k)} .
\end{aligned}
$$


One deduces that

$$
\begin{aligned}
\sum_{k=1}^{m} A(k-1, \triangle u(k-1)) & \leq C_{1} \sum_{k=1}^{m}|\triangle u(k-1)|+C_{1} \sum_{k=1}^{m} \frac{|\triangle u(k-1)|^{p(k-1)}}{p(k-1)} \\
& \leq C_{1} \sum_{k=1}^{m}\left(1+|\triangle u(k-1)|^{p(k-1)}\right)+\frac{C_{1}}{p^{-}} \sum_{k=1}^{m}|\triangle u(k-1)|^{p(k-1)} \\
& \leq m C_{1}+\left(C_{1}+\frac{C_{1}}{p^{-}}\right) \sum_{k=1}^{m}|\triangle u(k-1)|^{p(k-1)} .
\end{aligned}
$$

Using the hypothesis $\left(H_{9}\right)$ and according to the above result we have

$$
\begin{aligned}
\widehat{M}\left(\sum_{k=1}^{m} A(k-1, \triangle u(k-1))\right) & \leq \frac{B_{2}}{\alpha}\left[\left(\sum_{k=1}^{m} A(k-1, \Delta u(k-1))\right)^{\alpha}\right] \\
& \leq \frac{B_{2}}{\alpha}\left[1+m C_{1}+\left(C_{1}+\frac{C_{1}}{p^{-}}\right) \sum_{k=1}^{m}|\Delta u(k-1)|^{p(k-1)}\right]^{\alpha} \\
& \leq \frac{B_{2}}{\alpha}\left[\left(1+m C_{1}\right)+\left(\left(C_{1}+\frac{C_{1}}{p^{-}}\right) \sum_{k=1}^{m}|\triangle u(k-1)|^{p(k-1)}\right)\right]^{\alpha} .
\end{aligned}
$$

As function $x \longmapsto x^{\alpha}$ being convex for all $\alpha>1$, then we have the inequality

$$
(a+b)^{\alpha} \leq 2^{\alpha-1}\left(a^{\alpha}+b^{\alpha}\right) \text {, for all } a, b \in \mathbb{R} .
$$

Consequently, we have

$$
\widehat{M}\left(\sum_{k=1}^{m} A(k-1, \triangle u(k-1))\right) \leq \frac{B_{2}}{\alpha} 2^{\alpha-1}\left[\left(1+m C_{1}\right)^{\alpha}+\left(C_{1}+\frac{C_{1}}{p^{-}}\right)^{\alpha}\left(\sum_{k=1}^{m}|\triangle u(k-1)|^{p(k-1)}\right)^{\alpha}\right] .
$$

Using the relation $\left(a_{3}\right)$ we get

$$
\begin{aligned}
\widehat{M}\left(\sum_{k=1}^{m} A(k-1, \triangle u(k-1))\right) & \leq \frac{B_{2}}{\alpha} 2^{\alpha-1}\left[\left(1+m C_{1}\right)^{\alpha}+\left(C_{1}+\frac{C_{1}}{p^{-}}\right)^{\alpha} m^{\alpha}\left(2^{p^{+}}\|u\|_{e}^{p^{+}}+1\right)^{\alpha}\right] \\
& \leq \frac{B_{2}}{\alpha} 2^{\alpha-1}\left[\left(1+m C_{1}\right)^{\alpha}+\left(C_{1}+\frac{C_{1}}{p^{-}}\right)^{\alpha} m^{\alpha} 2^{\alpha-1}\left(2^{\alpha p^{+}}\|u\|_{e}^{\alpha p^{+}}+1\right)\right] \\
& \leq \frac{B_{2}}{\alpha} 2^{\alpha-1}\left(1+m C_{1}\right)^{\alpha}+\frac{B_{2}}{\alpha}\left(C_{1}+\frac{C_{1}}{p^{-}}\right)^{\alpha} m^{\alpha} 2^{2 \alpha-2} \\
& +\frac{B_{2}}{\alpha}\left(C_{1}+\frac{C_{1}}{p^{-}}\right)^{\alpha} m^{\alpha} 2^{2 \alpha-2+\alpha p^{+}}\|u\|_{e}^{\alpha p^{+}} .
\end{aligned}
$$

According to $\left(a_{4}\right)$, we have

$$
F(k, u) \geq \alpha_{1}(k)|u|^{s(k)}+\alpha_{2}(k) .
$$

Consequently,

$$
-\lambda \sum_{k=1}^{m} F(k, u) \leq-\lambda \sum_{k=1}^{m}\left(\alpha_{1}(k)|u(k)|^{s(k)}+\alpha_{2}(k)\right) .
$$


Using $\left(a_{2}\right)$ we obtain

$$
-\lambda \sum_{k=1}^{m} F(k, u) \leq-\lambda \alpha_{1}^{-} m^{\frac{2-s^{-}}{2}}\|u\|_{e}^{s^{-}}-\lambda \alpha_{2}^{-} m .
$$

Finally we get

$$
\begin{aligned}
J_{m}(u) & \leq \frac{B_{2}}{\alpha} 2^{\alpha-1}\left(1+m C_{1}\right)^{\alpha}+\frac{B_{2}}{\alpha}\left(C_{1}+\frac{C_{1}}{p^{-}}\right)^{\alpha} m^{\alpha} 2^{2 \alpha-2}+\frac{B_{2}}{\alpha}\left(C_{1}+\frac{C_{1}}{p^{-}}\right)^{\alpha} m^{\alpha} 2^{2 \alpha-2+\alpha p^{+}}\|u\|_{e}^{\alpha p^{+}} \\
& -\lambda \alpha_{1}^{-} m^{\frac{2-s^{-}}{2}}\|u\|_{e}^{s^{-}}-\lambda \alpha_{2}^{-} m .
\end{aligned}
$$

Since $s^{-}>\alpha p^{+}$, we see that $J_{m}$ is anti-coercive on $H_{m}$.

Lemma 5.3. Assume that conditions $\left(H_{1}\right),\left(H_{2}\right),\left(H_{9}\right)$ and $\left(a_{4}\right)$ hold with $s^{-}=\alpha p^{+}$. Then the functional $J_{m}$ is anti-coercive on $H_{m}$, for any $\lambda \in\left(\lambda_{0},+\infty\right)$, where

$$
\lambda_{0}=\frac{(2 m)^{2 \alpha-2+s^{-}} B_{2} C_{1}^{\alpha}}{\alpha_{1}^{-} \alpha}\left(\frac{p^{-}+1}{p^{-}}\right)^{\alpha} .
$$

\section{Proof.}

Based on the proof of Lemma 5.2, we get

$$
\begin{aligned}
J_{m} & \leq\left[\frac{B_{2}}{\alpha}\left(C_{1}+\frac{C_{1}}{p^{-}}\right)^{\alpha} m^{\alpha} 2^{2 \alpha-2+\alpha p^{+}}-\lambda \alpha_{1}^{-} m^{\frac{2-s^{-}}{2}}\right]\|u\|_{e}^{s^{-}}+\frac{B_{2}}{\alpha} 2^{\alpha-1}\left(1+m C_{1}\right)^{\alpha} \\
& +\frac{B_{2}}{\alpha}\left(C_{1}+\frac{C_{1}}{p^{-}}\right)^{\alpha} m^{\alpha} 2^{2 \alpha-2}-\lambda \alpha_{2}^{-} m .
\end{aligned}
$$

Thus, $J_{m}$ is anti-coercive on $H_{m}$ for any $\lambda \in\left(\lambda_{0},+\infty\right)$.

Note that for any $p^{+} \geq 1$, the functional $\|\cdot\|_{p^{+}}: Y_{a} \longrightarrow \mathbb{R}$ defined by

$$
\|u\|_{p^{+}}:=\left(\sum_{k=1}^{m}|\Delta u(k-1)|^{p^{+}}\right)^{\frac{1}{p^{+}}}
$$

is a norm on $Y_{a}$, while it is obviously not a norm on $H_{m}$. Since all norms on $Y_{a}$ are equivalent, therefore there exists a constant $\zeta>0$ such that

$$
\sum_{k=1}^{m}|\triangle u(k-1)|^{p^{+}} \geq \zeta\|u\|_{e}^{p^{+}},
$$

for all $u \in Y_{a}$.

Theorem 5.4. Assume that conditions $\left(H_{1}\right)-\left(H_{9}\right)$ and $\left(a_{4}\right),\left(a_{5}\right),\left(a_{6}\right)$ hold with $s^{-}>\alpha p^{+}$. Let $\lambda>0$ be fixed. Then problem (1.1) has at least three m-periodic solutions, at least two of which are non-trivial. 


\section{Proof.}

Choose a positive real number $\epsilon$ satisfying

$$
\epsilon \leq \frac{B_{1} \zeta^{\alpha}}{\alpha \lambda m\left(p^{+}\right)^{\alpha}}
$$

By $\left(a_{5}\right)$ and $\left(a_{6}\right)$ there exists $\rho \in\left(0, \omega_{0}\right)$ with $\omega_{0}<\frac{1}{4}$ such that

$$
F(k, u) \leq \epsilon|u|^{s^{-}} \text {for }|u| \leq \rho .
$$

If $u \in Y_{a}$ with $\|u\| \leq \rho$ then $|u(k)| \leq \rho$ for all $k \in \mathbb{Z}$.

From hypothesis $\left(H_{4}\right)$ and $\left(H_{9}\right)$,

$$
\begin{aligned}
\widehat{M}\left(\sum_{k=1}^{m} A(k-1, \Delta u(k-1))\right) & \geq \frac{B_{1}}{\alpha}\left(\sum_{k=1}^{m} A(k-1, \Delta u(k-1))\right)^{\alpha} \\
& \geq \frac{B_{1}}{\alpha}\left(\frac{1}{p(k-1)} \sum_{k=1}^{m}|\Delta u(k-1)|^{p(k-1)}\right)^{\alpha} \\
& \geq \frac{B_{1}}{\alpha}\left(\frac{1}{p^{+}} \sum_{k=1}^{m}|\Delta u(k-1)|^{p^{+}}\right)^{\alpha} .
\end{aligned}
$$

By using (5.1), it follows that

$$
\widehat{M}\left(\sum_{k=1}^{m} A(k-1, \Delta u(k-1))\right) \geq \frac{B_{1}}{\alpha\left(p^{+}\right)^{\alpha}} \zeta^{\alpha}\|u\|_{e}^{\alpha p^{+}} .
$$

By using (5.2) we have

$$
-\lambda \sum_{k=1}^{m} F(k, u) \geq-\lambda \epsilon \sum_{k=1}^{m}|u(k)|^{s^{-}} .
$$

Consequently,

$$
\begin{aligned}
J_{m}(u) & \geq \frac{B_{1}}{\alpha\left(p^{+}\right)^{\alpha}} \zeta^{\alpha}\|u\|_{e}^{\alpha p^{+}}-\lambda \epsilon \sum_{k=1}^{m}|u(k)|^{s^{-}} \\
& \geq \frac{B_{1}}{\alpha\left(p^{+}\right)^{\alpha}} \zeta^{\alpha}\|u\|_{e}^{\alpha p^{+}}-\lambda \epsilon m\|u\|_{e}^{s^{-}} \\
& \geq \frac{B_{1}}{\alpha\left(p^{+}\right)^{\alpha}} \zeta^{\alpha}\|u\|_{e}^{s^{-}}-\lambda \epsilon m\|u\|_{e}^{s^{-}} \\
& \geq\|u\|_{e}^{s^{-}}\left(\frac{B_{1}}{\alpha\left(p^{+}\right)^{\alpha}} \zeta^{\alpha}-\lambda \epsilon m\right) \geq 0 .
\end{aligned}
$$


Thus, the above relation together with $\left(H_{1}\right)$ and $\left(H_{8}\right)$ yields

$$
J_{m}(u) \geq J_{m}(0) \text { for all } u \in Y_{a} \text { with }\|u\|_{e} \leq \rho .
$$

Note also that for every $u \in W_{a}$ we have $\Delta u(k-1)=0$ for all $k \in \mathbb{Z}$ and then, according to $\left(H_{1}\right), A(k, 0)=0 ;$ so,

$$
J_{m}(u)=-\lambda \sum_{k=1}^{m} F(k, u(k)),
$$

for all $u \in W_{a}, \forall a \in \mathbb{R}^{n}$. If $u \in W_{a}$ with $\|u\|_{e} \leq \rho$ then $|u(k)| \leq \omega_{0}$ for all $k \in \mathbb{Z}$. Thus, by $\left(a_{4}\right)$ and $\left(H_{8}\right)$ it follows that

$$
J_{m}(u) \leq J_{m}(0) \text { for all } u \in W_{a} \text { with }\|u\|_{e} \leq \rho \text {, where } a \in \mathbb{R}^{n} .
$$

Let $\Psi_{m}=-J_{m}$. Using Lemma 5.2 we deduce that $\Psi_{m}$ satisfies the (PS) condition. Note that $\Psi_{m}$ is bounded from below, moreover as $\Psi_{m}$ is coercive and continuous it admits a minimizer. Using $\left(b_{2}\right)$ we obtain

$$
\inf _{u \in H_{m}} \Psi(u)=-\sup _{u \in H_{m}} J(u)<0 .
$$

We have shown that assumptions of Theorem 2.2 are satisfied, so $\Psi_{m}$ has at least three critical points, at least two of them are non-zero critical points. By Lemma 4.3 these are non-trivial $m$-periodic solutions of problem (1.1).

In the case $s^{-}=\alpha p^{+}$we have the following special case.

Corollary 5.5. Assume that conditions $\left(H_{1}\right)-\left(H_{9}\right)$ and $\left(a_{4}\right),\left(a_{5}\right),\left(a_{6}\right)$ hold with $s^{-}=p^{+}$. Let $\lambda \in\left(\lambda_{0},+\infty\right)$. Then, problem (1.1) has at least three m-periodic solutions, at least two of which are non-trivial.

\section{Multiple solutions of problem (1.1) by three critical points theorem}

In this pragraph we use the Theorem 3.3 to show the existence of multiple solutions for the problem (1.1).

Let functionals $\mu, J: H_{m} \longrightarrow \mathbb{R}$ be defined by

$$
\mu(u):=\widehat{M}\left(\sum_{k=1}^{m} A(k-1, \Delta u(k-1))\right) \text { and } J(u):=-\sum_{k=1}^{m} F(k, u(k)) .
$$

Then, we see that $J_{m}=\mu+\lambda J$.

Assume that $F$ has the following properties. 
$\left(H_{10}\right)$ : There exists a constant $C \in \mathbb{R}$ such that

$$
F(k, u) \leq C \text { for all }(k, u) \in \mathbb{Z} \times \mathbb{R}^{n}
$$

$\left(H_{11}\right)$ : There exist numbers $\tau_{1}, \tau_{2}, \tau_{3}>0, \tau_{3}>\tau_{2}>\tau_{1}$ such that for all $k \in \mathbb{Z}$ :

$\left(H_{11}^{\prime}\right) F(k, u)<0$ with $0<|u| \leq \tau_{1}$,

$\left(H_{11}^{\prime \prime}\right) F(k, u)>0$ with $\tau_{2} \leq|u| \leq \tau_{3}$.

Example 6.1. As example of function satisfying assumptions $\left(H_{6}\right)-\left(H_{8}\right)$ and $\left(H_{10}\right)-\left(H_{11}\right)$ we can give the following, where $m \geq 2$ is a fixed even natural number.

$F: \mathbb{Z} \times \mathbb{R}^{n}$ given by

$$
F(k, t):=\left\{\begin{array}{l}
-\sin |t| \text { if }|t| \leq \pi, \\
|t-\pi| \text { if } \pi<|t|<2 \pi, \\
\pi \text { if }|t| \geq 2 \pi .
\end{array}\right.
$$

In this pragraph we will consider the problem (1.1) on $Y_{a}$.

Remark 6.2. The functional $\mu$ is not coercive on $H_{m}$, but it is coercive on $Y_{a}$, for $a \in \mathbb{R}^{n}$. Indeed, given a sequence $\left(u_{n}\right)_{n \in \mathbb{N}}$ such that $u_{n}(k)=a$ for all $n \in \mathbb{N}$ and all $k \in \mathbb{Z}$, we see that

$$
\mu\left(u_{n}\right)=\widehat{M}\left(\sum_{k=1}^{m} A\left(k-1, \Delta u_{n}(k-1)\right)\right)=\widehat{M}\left(\sum_{k=1}^{m} A(k-1,0)\right)=0 .
$$

Letting $a \longrightarrow+\infty$, we see that $\mu$ is not coercive on $H_{m}$. Furthermore, by using (5.3) we get

$$
\widehat{M}\left(\sum_{k=1}^{m} A(k-1, \Delta u(k-1))\right) \geq \frac{B_{1}}{\alpha\left(p^{+}\right)^{\alpha}} \zeta^{\alpha}\|u\|_{e}^{\alpha p^{+}} .
$$

It is inferred that $\mu$ is coercive on $Y_{a}$.

Let us note that the solutions obtained being in $Y_{a}$, for $a \in \mathbb{R}^{n}$; there are therefore no constant solutions, however there may still be a zero solution.

Theorem 6.3. Assume that conditions $\left(H_{1}\right)-\left(H_{11}\right)$ hold. Then there exists $\lambda>0$ such that problem (1.1) has at least three solutions in $Y_{a}$, at least two of which are necessarily non-zero.

\section{Proof.}

Based on previous results, $\mu$ is coercive on $Y_{a}$ and is $C^{1}$, therefore, $\mu$ verifies assumptions of 
Theorem 3.3.

According to the hypothesis $\left(H_{10}\right)$,

$$
F(k, u(k)) \leq C \Longrightarrow J(u) \geq-m C>-\infty .
$$

Hence $J$ is bounded from below.

By $\left(H_{11}^{\prime \prime}\right)$ it follows that

$$
-F(k, u(k))<0,
$$

for all $u \in Y_{a}$ such that $\tau_{2} \leq|u(k)| \leq \tau_{3}$ for all $k \in \mathbb{Z}$. We thus deduce that there exists a point $u \in Y_{a}$ such that $J(u)<0$. Consequently,

$$
\inf _{u \in Y_{a}} J(u)<0
$$

By $\left(H_{11}^{\prime}\right)$ and since $\mu$ is continuous, coercive, convex, non-negative and $\mu(0)=0$, we get that there are $s, r>0$, such that $J(u)>0$ for $r \leq \mu(u) \leq s$. Therefore, $\left(b_{2}\right)$ is satisfied.

Now, by $\left(H_{8}\right)$ and $\left(H_{11}^{\prime \prime}\right)$ we obtain

$$
J(0)=0<\inf _{r \leq \mu(u) \leq s} J(u) .
$$

Hence, condition $\left(b_{3}\right)$ is satisfied. Thus, by Theorem 3.3 we see that there exists $\lambda>0$ such that the functional $J_{m}$ has at least three critical points on $Y_{a}$. Since by Lemma 4.3 critical points of $J_{m}$ are solutions of problem (1.1), hence the result follows.

For numbers $r_{0}, s_{0}>0$ we put

$$
r^{\prime}:=\inf \left\{\|u\|_{\max }: \mu(u) \geq r_{0}\right\} \text { and } s^{\prime}:=\sup \left\{\|u\|_{\text {max }}: \mu(u) \leq s_{0}\right\},
$$

where

$$
\|u\|_{\max }=\max _{k \in \mathbb{Z}[1, m]}|u(k)|
$$

Hence,

$$
\left\{u \in Y_{a}: \mu(u) \geq r_{0}\right\} \subset\left\{u \in Y_{a}:\|u\|_{\max } \geq r^{\prime}\right\}
$$

and

$$
\left\{u \in Y_{a}: \mu(u) \leq s_{0}\right\} \subset\left\{u \in Y_{a}:\|u\|_{\max } \leq s^{\prime}\right\} .
$$

Assume that $F$ satisfies the following.

$\left(H_{12}\right)$ : There exist constants $r_{0}, s_{0}>0, r_{0}<s_{0}$ such that the following relations hold

$$
\left(H_{12}^{\prime}\right) \sup _{|u|<s^{\prime}} F\left(k_{0}, u\right)<\sup _{u \in \mathbb{R}^{n}} F(k, u) \text { for some } k_{0} \in \mathbb{Z}[1, m]
$$

$$
\left(H_{12}^{\prime \prime}\right) F(k, u) \leq 0 \text { for all }(k, u) \in \mathbb{Z}[1, m] \times \mathbb{R}^{n} \text { with } r^{\prime} \leq|u| \leq s^{\prime} .
$$


Example 6.4. As examples of functions satisfying assumptions $\left(H_{6}\right)-\left(H_{8}\right),\left(H_{10}\right)$ and $\left(H_{12}\right)$ we can give the following, where $m \geq 2$ is a fixed even natural number.

(1) $F: \mathbb{Z} \times \mathbb{R}^{n}$ given by

$$
F(k, t):=\left\{\begin{array}{l}
\frac{3}{5} \pi|t| \text { if }|t| \leq \frac{5}{6} \pi \\
\sin |t| \text { if } \frac{5}{6} \pi<|t|<2 \pi \\
\sin |t| a(k) \text { if }|t| \geq 2 \pi
\end{array}\right.
$$

where $a: \mathbb{Z} \rightarrow \mathbb{R}$ is defined by

$$
a(k):=\left\{\begin{array}{l}
\frac{1}{2} \text { if } k=2 l, \\
1 \text { if } k=2 l+1 ; l \in \mathbb{Z} .
\end{array}\right.
$$

Remark 6.5. Note that $\left(H_{12}^{\prime}\right)$ is equivalent to the following hypothesis.

$$
\sum_{k=1}^{m} \sup _{|t| \leq s^{\prime}} F(k, t)<\sum_{k=1}^{m} \sup _{t \in \mathbb{R}} F(k, t) ;
$$

in fact from $(6.1)$ it is easy to get $\left(H_{11}^{\prime}\right)$, since

$$
\sup _{|t| \leq s^{\prime}} F(k, t) \leq \sup _{t \in \mathbb{R}} F(k, t) \text { for any } s^{\prime}>0 \text { and for any } k \in \mathbb{Z}[1, m] .
$$

On the other hand, since (6.1) is equivalent to the following

$$
\left(\sup _{t \in \mathbb{R}} F(1, t)-\sup _{|t|<s^{\prime}} F(1, t)\right)+\left(\sup _{t \in \mathbb{R}} F(2, t)-\sup _{|t|<s^{\prime}} F(2, t)\right)+\ldots+\left(\sup _{t \in \mathbb{R}} F(m, t)-\sup _{|t|<s^{\prime}} F(m, t)\right)>
$$

0, we get (6.1), noting again (6.2). Assumption (6.1) was used in the context of anisotropic problems in [13].

Theorem 6.6. Let $r_{0}, s_{0}>0, r_{0}<s_{0}$. Assume that conditions $\left(H_{1}\right)-\left(H_{10}\right)$ and $\left(H_{12}\right)$ hold. Then, there exists $\lambda>0$ such that problem (1.1) has at least three solutions on $Y_{a}$, at least two of which are non-trivial.

\section{Proof.}

We will show that $J_{m}$ verifies assumptions of Theorem 3.3. Clearly, $\mu$ is coercive and is of class $C^{1}$ on $Y_{a}, \mu(0)=0$ and $J$ is bounded from below. By $\left(H_{12}^{\prime}\right)$, we deduce that

$$
\begin{aligned}
\inf _{u \in Y_{a}} J(u) & =-\sum_{k=1}^{m} \sup _{\left\{i \in \mathbb{Z}[1, m]: u(i) \in \mathbb{R}^{n}\right\}} F(k, u(i)) \\
& <-\sum_{k=1}^{m} \sup _{\left\{i \in \mathbb{Z}[1, m]: u(i) \leq s^{\prime}\right\}} F(k, u(i))=\inf _{\|u\|_{\text {max }} \leq s^{\prime}} J(u) \leq \inf _{\mu(u) \leq s_{0}} J(u) .
\end{aligned}
$$


Thus, $\left(b_{2}\right)$ is satisfied. For any $u \in Y_{a}$ with $r_{0} \leq \mu(u) \leq s_{0}$, we have $r^{\prime} \leq\|u\|_{\text {max }} \leq s^{\prime}$. By $\left(H_{12}^{\prime \prime}\right)$, we obtain

$$
\begin{aligned}
\inf _{r_{0} \leq \mu(u) \leq s_{0}} J(u) & \geq \inf _{r^{\prime} \leq\|u\|_{\text {max }} \leq s^{\prime}} J(u) \\
& =-\sum_{k=1}^{m}\left\{i \in \mathbb{Z}[1, m]: r^{\prime} \leq \max _{i \in \mathbb{Z}[1, m]}|u(i)| \leq s^{\prime}\right\}
\end{aligned}
$$

Therefore $\left(b_{3}\right)$ is satisfied. Finally, by Theorem 3.3 there exists $\lambda>0$ such that the functional $J_{m}$ has at least three critical points in $Y_{a}$. Consequently, by Lemma 4.3, problem (1.1) has at least three solutions. Note that without the hypothesis $\left(H_{12}^{\prime}\right)$ it is possible to obtain a type of small solutions, lying near the origin.

Let us consider the following remark.

Remark 6.7 . Let $k_{0} \in \mathbb{Z}[1, m]$ be a fixed real. Since $F\left(k_{0}, t\right) \leq C$ for some $C>0$, so

$$
\sup _{t \in \mathbb{R}^{n}} F\left(k_{0}, t\right)=\beta \leq C .
$$

If $\beta>0$ we can reason as follows. Since $t \longmapsto F\left(k_{0}, t\right)$ is continuous and since $F\left(k_{0}, 0\right)=0$, there exists sufficiently small positive $s^{\prime}$ depending on $\beta$ such that

$$
-\frac{\beta}{2} \leq F\left(k_{0}, t\right) \leq \frac{\beta}{2} \text {. }
$$

Thus,

$$
\sup _{|t| \leq s^{\prime}} F\left(k_{0}, t\right)<\sup _{t \in \mathbb{R}^{n}} F\left(k_{0}, t\right)
$$

From the above remark we have the following result.

Corollary 6.8. Assume that conditions $\left(H_{1}\right)-\left(H_{10}\right)$ and $\left(H_{12}^{\prime \prime}\right)$ hold. Let

$$
\sup _{t \in \mathbb{R}^{n}} F\left(k_{0}, t\right)>0,
$$

for some $k_{0} \in \mathbb{Z}[1, m]$. Then, there exists $\lambda>0$ such that problem (1.1) has at least three solutions on $Y_{a}$, for $a \in \mathbb{R}^{n}$, at least two of which are non-trivial.

We can also replace $\left(H_{12}^{\prime \prime}\right)$ with $\left(H_{11}^{\prime}\right)$ provided that $\tau_{1}$ is sufficiently large. Indeed, we can reason as follows.

Remark 6.9. Let $s_{0}>\frac{B_{1} \zeta^{\alpha}}{\alpha\left(p^{+}\right)^{\alpha}}, 0<r_{0}<s_{0}$ and $\tau_{0}=\zeta_{0}\left[\frac{s_{0} \alpha\left(p^{+}\right)^{\alpha}}{B_{1} \zeta^{\alpha}}\right]^{\frac{1}{\alpha p^{+}}}$. If $\left(H_{11}^{\prime}\right)$ is satisfied with $\tau_{1} \geq \tau_{0}$, then $\left(H_{12}^{\prime \prime}\right)$ is also satisfied with $s^{\prime}=\tau_{0}$. The inequality $\mu(u) \leq s_{0}$ is equivalent 
to

$$
\widehat{M}\left(\sum_{k=1}^{m} A(k-1, \Delta u(k-1))\right) \leq s_{0}
$$

Using (5.3) we obtain $\frac{B_{1}}{\alpha\left(p^{+}\right)^{\alpha}} \zeta^{\alpha}\|u\|_{e}^{\alpha p^{+}} \leq s_{0}$. Consequently, since $s_{0}>\frac{B_{1} \zeta^{\alpha}}{\alpha\left(p^{+}\right)^{\alpha}}$, we get

$$
\|u\|_{e} \leq\left[\frac{s_{0} \alpha\left(p^{+}\right)^{\alpha}}{B_{1} \zeta^{\alpha}}\right]^{\frac{1}{\alpha p^{+}}}
$$

Since all norms on $Y_{a}$, for $a \in \mathbb{R}^{n}$ are equivalent, therefore, there exists a constant $\zeta_{0}>0$ such that $\|u\|_{\max } \leq \zeta_{0}\|u\|_{e}$; hence

$$
\|u\|_{\max } \leq \zeta_{0}\left[\frac{s_{0} \alpha\left(p^{+}\right)^{\alpha}}{B_{1} \zeta^{\alpha}}\right]^{\frac{1}{\alpha p^{+}}}
$$

and

$$
s^{\prime}=\sup \left\{\|u\|_{\text {max }}: \mu(u) \leq s_{0}\right\}=\zeta_{0}\left[\frac{s_{0} \alpha\left(p^{+}\right)^{\alpha}}{B_{1} \zeta^{\alpha}}\right]^{\frac{1}{\alpha p^{+}}}
$$

Furthermore

$$
\left\{u \in Y_{a}: \mu(u) \leq s_{0}\right\} \subset\left\{u \in Y_{a}:|u| \leq \zeta_{0}\left[\frac{s_{0} \alpha\left(p^{+}\right)^{\alpha}}{B_{1} \zeta^{\alpha}}\right]^{\frac{1}{\alpha p^{+}}}\right\} \subset\left\{u \in Y_{a}:|u| \leq \tau_{1}\right\}
$$

Corollary 6.10. Let $s_{0}>\frac{B_{1} \zeta^{\alpha}}{\alpha\left(p^{+}\right)^{\alpha}}, 0<r_{0}<s_{0}$ and $\tau_{0}=\zeta_{0}\left[\frac{s_{0} \alpha\left(p^{+}\right)^{\alpha}}{B_{1} \zeta^{\alpha}}\right]^{\frac{1}{\alpha p^{+}}}$. Assume that conditions $\left(H_{1)}-\left(H_{10}\right)\right.$ and $\left(H_{12}^{\prime}\right)$ hold. If moreover $\left(H_{11}^{\prime}\right)$ is satisfied with $\tau_{1} \geq \tau_{0}$, then there exists $\lambda>0$ such that problem (1.1) has at least three solutions on $Y_{a}$, for $a \in \mathbb{R}^{n}$, at least two of which are non-trivial.

Analogously we obtain the following.

Corollary 6.11. Let $s_{0} \leq \frac{B_{1} \zeta^{\alpha}}{\alpha\left(p^{+}\right)^{\alpha}}, \quad 0<r_{0}<s_{0}$ and $\tau_{0}=\zeta_{0}\left[\frac{s_{0} \alpha\left(p^{+}\right)^{\alpha}}{B_{1} \zeta^{\alpha}}\right]^{\frac{1}{\alpha p^{-}}}$. Assume that conditions $\left(H_{1}\right)-\left(H_{10}\right)$ and $\left(H_{12}^{\prime}\right)$ hold. If moreover $\left(H_{11}^{\prime}\right)$ is satisfied with $\tau_{1} \geq \tau_{0}$, then there exists $\lambda>0$ such that problem (1.1) has at least three solutions on $Y_{a}$, for $a \in \mathbb{R}^{n}$, at least two of which are non-trivial. 


\section{REFERENCES}

[1] R.P. Agarwal, K. Perera, D. ORegan, Multiple positive solutions of singu- lar and nonsingular discrete problems via variational methods, Nonlinear Anal., 58 (2004), 69-73.

[2] R.P. Agarwal, K. Perera, D. ORegan, Multiple positive solutions of singu- lar discrete p-Laplacian problems via variational methods, Adv. Diff. Equ., 2 (2005), 93-99.

[3] G. Bonanno, P. Candito, Nonlinear difference equations investigated via critical point methods, Nonlinear Ana, 70 (2009), 3180-3186.

[4] A. Cabada, A. Lannizzotto, S. Tersian, Multiple solutions for discrete boundary value problems, J. Math. Anal. Appl., 356 (2009), 418-428.

[5] P. Candito, N. Giovannelli, Multiple solutions for a discrete boundary value problem involving the p-Laplacian, Math. Appl. Comput., 56 (2008), 959- 964.

[6] X. Cai, J. Yu, Existence theorems for second-order discrete boundary value problems, J. Math. Anal. Appl., 320 (2006), 649-661.

[7] G. F. Carrier; A note on the vibrating string, Quart. Appl. Math. 7 (1949), 97-101.

[8] G. F. Carrier; On the nonlinear vibration problem of the elastic string, Quart. Appl. Math. 3 (1945), 157-165.

[9] K.-C. F. Chang; Variational methods for non-differentiable functionals and their applications to partial differential equations, J. Math. Anal. Appl. 80(1) (1981), pp. 102-129.

[10] F.H. Clarke; Optimization and Nonsmooth Analysis, Classics in Applied Mathematics, Vol. 5, SIAM, Philadelphia, 1990.

[11] M. Galewski, G. M. Bisci and R. Wieteska; Existence and multiplicity of solutions to discrete inclusions with the $p(k)$-Laplacian problem, J.Difference Equ.Appl.21, No. 10(2015), 887-903.

[12] M. Galewski and R. Wieteska; Multiple solutions for periodic problems with the discrete $p(k)$-Laplacian, Discrete Contin. Dyn. Syst. Ser. B 19(8) (2014), pp. 2535-2547.

[13] M. Galewski and S. Glab; On the discrete boundary value problem for anisotropic equation, Math. Anal. Appl. 386(2) (2012), pp. 956-965. 
[14] A. Guiro, I. Nyanquini, S. Ouaro, On the solvability of discrete nonlin- ear Neumann problems involving the $p(x)$-Laplacian, Adv. Diff. Equ., 32 (2011).

[15] P. Jebelean, C. Serban, Ground state periodic solutions for difference equa- tions with discrete p-Laplacian, Appl. Math. Comput., 217 (2011), 9820- 9827.

[16] G. Kirchhoff; Vorlesungen uber mathematische Physik: Mechanik, Teubner, Leipzig, 1876.

[17] B. Koné, I. Nyanquini and S. Ouaro; Weak solutions to discrete nonlinear two-point boundary-value problems of Kirchhoff type, Electronic Journal of Differential Equations, Vol. 2015 (2015), No. 105, pp. $1-10$.

[18] B. Kone, S. Ouaro, Weak solutions for anisotropic discrete boundary value problems, J. Diff. Equ. Appl. (2010), 1-11.

[19] A. Kristaly, V. Radulescu and Cs Varga; Variational Principles in Mathematical Physics, Geometry, and Economics: Qualitative Analysis of Nonlinear Equations and Unilateral Problems, Encyclopedia of Mathematics and Its Applications, No. 136, Cambridge University Press, Cambridge, 2010.

[20] R.A. Mashiyev, Z. Yucedag, S. Ogras, Existence and multiplicity of solu- tions for a Dirichlet problem involving the discrete $p(x)$-Laplacian operator, E.J. Qualitative Theory of Diff. Equ., 67 (2011), 1-10.

[21] M. Mihailescu, V. Radulescu, S. Tersian, Eigenvalue problems for anisotropic discrete boundary value problems, J. Difference Equ. Appl., 15 (2009), 557-567.

[22] D. Motreanu and V. Radulescu; Variational and Non-Variational Methods in Nonlinear Analysis and Boundary Value Problems, Nonconvex Optimization and Its Applications, Vol. 67, Kluwer Academic Publishers, Dordrecht, 2003.

[23] D. Motreanu and Cs Varga; Some critical point result for locally Lipschitz functionals, Comm. Appl. Nonlinear Anal. 4 (1997), pp. 17-33.

[24] R. Narashima; Nonlinear vibration of an elastic string, J. Sound Vibration 8 (1968), 134-146.

[25] D. W. Oplinger; Frequency response of a nonlinear stretched string, J. Acoustic Soc. Amer. 32(1960), 1529-1538.

[26] X. Wu; A new critical point theorem for locally Lipschitz functionals with applications to differential equations, Nonlinear Anal. 66(3) (2007), pp. 624-638. 
[27] J. Yu, Z. Guo, On boundary value problems for a discrete generalized Emden-Fowler equation, J. Math. Anal. Appl., 231 (2006), 18-31.

[28] Z. Yucedag; Existence of solutions for anisotropic discrete boundary value problems of Kirchhoff type,1364 (2014), 1-15.

[29] G. Zhang, S. Liu, On a class of semipositone discrete boundary value prob- lem, J. Math. Anal. Appl., 325 (2007), 175-182. 